\title{
Clinical value of a serum anti-PLA2R antibody in the diagnosis and monitoring of primary membranous nephropathy in adults
}

This article was published in the following Dove Press journal: International Journal of Nephrology and Renovascular Disease

\section{Xueping $\mathrm{Wu}^{\prime}$ \\ Lei Liu' \\ Yaling Guo' \\ Lijuan Yang ${ }^{2}$}

'Department of Nephrology, The First Affiliated Hospital of Bengbu Medical College, Bengbu, Anhui, People's Republic of China; ${ }^{2}$ Department of Physiology, Bengbu Medical College, Bengbu, Anhui, People's Republic of China
Correspondence: Lijuan Yang Department of Physiology, Bengbu Medical College, 2600 Donghai Road, Bengbu, Anhui 233030, People's Republic of China

Tel +86 I809655 99II

Email bbmcylj@163.com
Objective: To compare the positive rate of anti-PLA2R antibodies in patients with primary membranous nephropathy (PMN), secondary membranous nephropathy (SMN), and non-membrane nephropathy (non-MN); evaluate serum anti-PLA2R antibodies in the diagnosis of PMN; quantify the serum anti-PLA2R antibody levels during the treatment of PMN patients; and evaluate the clinical value of monitoring changes in serum anti-PLA2R antibody quantification levels. Methods: The kidney tissue was collected by kidney biopsy. The expression of PLA2R in glomeruli was detected by immunofluorescence, and ELISA was used to quantify the serum anti-PLA2R antibody. The positive rate of PLA2R expression in renal tissue and positive rate of the anti-PLA2R antibody in the three groups were compared and calculated using a statistical method. The specificity and coincidence rate of anti-PLA2R used in the differential diagnosis of PMN and SMN were evaluated. The clinical value of monitoring changes in serum anti-PLA2R antibody quantification levels was evaluated.

Result: The serum levels of the anti-PLA2R antibody were significantly higher in patients with PMN than in patients with SMN and non-MN group. The difference was statistically significant $(P<0.05)$. The serum anti-PLA2R antibody became negative in the complete remission group. The serum anti-PLA2R antibody levels were significantly lower than before treatment in the partial remission group, and the difference was statistically significant $(P<0.05)$. However, in the non-remission group, the serum anti-PLA2R antibody levels remained high.

Conclusion: Detection of the serum anti-PLA2R antibody has a high specificity for diagnosing PMN. The change of the serum anti-PLA2R antibody level is closely related to the status of the PMN: if the anti-PLA2R antibody level has decreased, it indicates that the condition has improved; and if the serum anti-PLA2R antibody continues to show high levels of positive or quantitative increase, the condition is not in remission or has relapsed.

Keywords: primary membranous nephropathy, phospholipase A2 receptor, serum anti-phospholipase $\mathrm{A} 2$ receptor antibody

\section{Introduction}

Membranous nephropathy (MN) refers to the recognition of target antigens of glomerular podocytes by autoantibodies in the circulation. After antigen binding to antibodies, immunological complex deposition is formed under epithelial cells, accompanied by glomerular basement membrane (GBM), a group of glomerular diseases characterized by diffuse thickening. ${ }^{1}$ Approximately $80 \%$ of $\mathrm{MNs}$ are primary $\mathrm{MN}(\mathrm{PMN})$, the remaining $20 \%$ are secondary to other systemic diseases or due to exposure to other factors, for example: infections, tumors, autoimmune diseases, drugs, etc. These diseases are known as secondary MN (SMN). ${ }^{2}$ With the populariza- 
tion of renal pathological diagnosis methods, most medical centers have reported that the incidence rates of $\mathrm{MN}$ are increasing year by year. ${ }^{3}$ Approximately one third of PMN patients have spontaneous remission, and one third to half of patients can progress to end-stage renal disease within 5-10 years if they are not diagnosed and treated promptly. ${ }^{4}$

PLA2R is an autoantigen present in glomerular podocytes. In 2009, Beck et $\mathrm{al}^{5}$ first discovered that the extracellular domain of PLA2R was used as the mutant antigen to activate autoimmune response. Combined with the antiPLA2R antibodies produced in the body, it forms the in situ immune complex that activates the complement system to cause podocyte injury, resulting in urine protein production, which is the major pathogenic factor of the majority of PMN patients. The serum PLA2R antibody has diagnostic specificity and can be used to distinguish between PMN and other glomerular diseases. ${ }^{6}$ It has been reported in People's Republic of China and other countries that serum anti-PLA2R antibody levels are associated with urinary protein levels, disease activity, improvement rate, recurrence, and drug resistance, etc. ${ }^{7-9}$ This study analyzed the positive rate of serum anti-PLA2R antibodies among the PMN group, the SMN group, and non-MN group; quantitative changes in serum anti-PLA2R antibody levels before and after treatment were measured. The value of serum anti-PLA2R antibodies in the diagnosis and monitoring of PMN and in evaluating the clinical significance of the treatment was explored.

\section{Objectives and methods}

\section{Research objects and grouping standards}

This study was approved by the Ethics Committee of the First Affiliated Hospital of Bengbu Medical College (Bengbu, People's Republic of China, 2016094). The study included 164 adult patients (including 88 males and 76 females) who underwent renal biopsy, selected from the Department of Nephrology, the First Affiliated Hospital of Bengbu Medical College, Bengbu, People's Republic of China, from January 2016 to July 2017. All patients were yellow race. All patients signed written informed consent. Inclusion criteria: 1) age $\geq 18$ years; 2) no use of glucocorticoids and immunosuppressive agents before renal biopsy; and 3) complete clinical data available.

The inclusion criteria for PMN were as follows: the pathological diagnosis of the kidney tissue was $\mathrm{MN}$, except for clinical factors such as systemic lupus erythematosus (SLE), hepatitis B virus (HBV), tumor, and drug-induced SMN. ${ }^{10}$

The inclusion criteria for SMN were as follows: 1) membranous lupus nephritis (MLN): $\geq$ SLE classification as revised by Systemic Lupus International Collaborating
Clinics in 2012 and the pathological diagnosis of the kidney tissue was MN; 2) HBV-related glomerulonephritis (HBV$\mathrm{GN}$ ): serum HBsAg was positive, except for clinical factors such as SLE, tumor, and drugs, the pathological diagnosis of the kidney tissue was $\mathrm{MN}$, and $\mathrm{HBsAg}$ and/or HBcAg in the kidney tissue was positive; and 3) atypical membranous nephropathy (AMN): in addition to GBM thickening, there were also mesangial cells and mesangial matrix hyperplasia under light microscopy; electron microscopy showed electron dense deposits in glomerular epithelial cells, basement membrane, subendothelial, and mesangial areas, etc; IgG, IgA, IgM, C3, C1q, etc were deposits in the form of granules or clumps along the glomerular capillary wall and mesangial area.

The inclusion criteria for non-MN were as follows: pathological findings did not meet $\mathrm{MN}$ criteria, including IgA nephropathy (IgAN), minimal change disease (MCD), mesangioproliferative glomerulonephritis (MsPGN), focal segmental glomerulosclerosis (FSGS), and membranoproliferative glomerulonephritis (MPGN).

According to this inclusion criteria, there were 64 cases in the PMN group, 40 cases in the SMN group (including 14 cases of MLN, eight cases of HBV-GN, and 18 cases of $\mathrm{AMN}$ ), and 60 cases in the non-MN group (including 22 cases of IgAN, 15 cases of MCD, 13 cases of MsPGN, seven cases of FSGS, and three cases of MPGN).

\section{Research methods \\ Clinical data}

Clinical data were collected from all patients, including age, gender, serum creatinine, serum albumin, 24-hour urinary protein, serum cholesterol, triglycerides, and serum anti-PLA2R antibody quantification. These indicators were reviewed each month after treatment in serum anti-PLA2R antibody-positive PMN patients and follow-up for 9 months.

\section{Renal histopathological examination}

Kidney tissue was collected from patients and the pathological specimens examined under light microscopy, immunofluorescence, and electron microscopy. The pathological specimens were sent to the Golden Medical Laboratory for evaluation.

\section{Renal tissue PLA2R examination was conducted using immunofluorescence}

Paraffin sections, with a thickness of $3 \mu \mathrm{m}$, were deparaffinized in water. The slides were then rinsed with PBS buffer. Digestion was conducted with $10 \%$ proteinase $\mathrm{K}$, and the slides 
were placed in an incubation box and incubated in a $37^{\circ} \mathrm{C}$ incubator for 10 minutes. The slides were washed with PBS buffer and the sections were kept moist. The PLA2R fluorescent antibody was added in a dropwise manner. The sections were incubated in a $37^{\circ} \mathrm{C}$ incubator in a wet incubation box for 60 minutes. The incubation process was repeated two times. Fluorescein isothiocyanate-labeled secondary antibody was added in a dropwise manner. The sections were placed in a wet incubator box and incubated in a $37^{\circ} \mathrm{C}$ incubator for 40 minutes. The slides were washed with PBS, mounted with neutral glycerol, and observed using a fluorescence microscope. Fluorescence intensity determination: observed by fluorescence microscopy, - , negative, $\pm /+\sim 3+$, positive. ${ }^{11}$

\section{Serum anti-PLA2R antibody quantification method} Using ELISA to reconstitute PLA2R as the antigen, the patient's serum served as the antibody to be detected, and peroxidase-labeled rabbit antihuman IgG antibody was the enzyme-labeled antibody. The assay was performed according to the manufacturer's instructions (EUROIMMUN, Lübeck, Germany) as follows: 1) the samples to be measured and the standards were prepared by adding the enzyme-labeled reagents and incubating for 1 hour at $37^{\circ} \mathrm{C}$; 2) the wells were washed five times with antiseptic solution after which the color solution was added and the plates were incubated in the dark at $37^{\circ} \mathrm{C}$ for 15 minutes; and 3) the stop solution was then added, and after the blue color changed to yellow, the absorbance of each well was measured with a microplate reader (Biotek, Winooski, VT, USA) at a wavelength of $450 \mathrm{~nm}$. The anti-PLA2R antibody (IgG) provided in the kit was used as a standard curve, and the four-parameter fitting equation was calculated. Antibody concentrations $<14$ $\mathrm{RU} / \mathrm{mL}$ were considered negative. Kits provide negative and positive controls.

\section{Follow-up index}

PMN patients who had anti-PLA2R antibody-positive serum were followed-up for 24-hour urinary protein, serum albumin, serum creatinine, and serum anti-PLA2R antibody quantitative levels. The patients were divided into complete remission, partial remission, and non-remission groups according to their condition after treatment. The PMN treatment mitigation standard was as follows: using the criteria developed by the 2012 Kidney Disease: Improving Global Outcomes Clinical Practice Guideline for Glomerulonephritis: ${ }^{12}$ 1) complete remission: 24-hour urinary protein quantification $<0.3 \mathrm{~g}$ and normal serum albumin and serum creatinine. 2)
Partial remission: 24-hour urinary protein quantification $<3.5 \mathrm{~g}$, and urinary protein excretion was reduced by $\geq 50 \%$. Serum albumin was normal or higher than before and serum creatinine was normal. 3) Non-remission: the previously mentioned mitigation criteria were no met.

\section{Statistical analysis}

Data were processed using SPSS 19.0 software. Normally distributed continuous variables were expressed as the mean \pm SD. Paired sample Student's $t$-test was used for comparison between the two groups. One-way ANOVA was used for comparison of more than two groups' data. Categorical variables were expressed as percentages, and single factor groups were compared using the chi-squared test or the Fisher's exact probability method. Differences were considered statistically significant at $P<0.05$.

\section{Results \\ General results}

According to the kidney pathology results, there were 64 cases in the PMN group, with an average age of $42 \pm 13$ years; 40 patients in the SMN group, aged $38 \pm 12$ years; and 60 patients in the non-MN group, aged $40 \pm 13$ years. There were no significant differences in clinical biochemical indexes among the three groups $(P>0.05)$ (Table 1$)$.

\section{Renal tissue PLA2R expression among the three groups of patients}

Immunofluorescence examination of glomerular PLA2Rpositive patients demonstrated 60 cases in the PMN group, accounting for $93.75 \%(60 / 64)$; ten cases in the SMN group, accounting for $25 \%(10 / 40)$; and 0 cases in the non-MN group (Figure 1).

The expression of PLA2R in glomeruli was significantly different among the three groups. Under immunofluorescence, deposition of a large number of immune complexes along the GBM in the PMN group, and deposition of a few or no immunolabeled complexes in the SMN group were observed. No deposition of immune complexes was observed in the non-MN group.

\section{Comparison of serum anti-PLA2R antibodies among the three groups}

The serum anti-PLA2R antibody levels in PMN, SMN, and non-MN groups were $226.62 \pm 366.49 \mathrm{RU} / \mathrm{mL}, 9.09 \pm 9.75 \mathrm{RU} /$ $\mathrm{mL}, 5.11 \pm 3.80 \mathrm{RU} / \mathrm{mL}$; and the antibody positive rates were $87.5 \%, 20 \%$, and $0 \%$, respectively (Tables 2 and 3 ). 
Table I Comparison of clinical biochemical indexes before treatment in three groups

\begin{tabular}{|l|l|l|l|l|l|l|}
\hline Groups & $\mathbf{n}$ & $\begin{array}{l}\text { Serum creatinine } \\
(\boldsymbol{\mu m o l} / \mathbf{L})\end{array}$ & $\begin{array}{l}\text { Serum albumin } \\
(\mathbf{g} / \mathbf{L})\end{array}$ & $\begin{array}{l}\text { 24-hour urinary } \\
\text { protein } \mathbf{( g )}\end{array}$ & $\begin{array}{l}\text { Blood cholesterol } \\
(\mathbf{m m o l} / \mathbf{L})\end{array}$ & $\begin{array}{l}\text { Blood triglyceride } \\
\mathbf{( m m o l} / \mathbf{L})\end{array}$ \\
\hline PMN & 64 & $65.44 \pm 12.06$ & $23.47 \pm 6.11$ & $5.67 \pm 1.70$ & $10.32 \pm 3.98$ & $2.99 \pm 1.72$ \\
SMN & 40 & $62.26 \pm 10.53$ & $25.38 \pm 5.79$ & $5.47 \pm 1.61$ & $9.28 \pm 3.37$ & $2.77 \pm 0.47$ \\
non-MN & 60 & $61.10 \pm 10.18$ & $24.00 \pm 5.58$ & $5.68 \pm 1.71$ & $10.07 \pm 3.01$ & $3.06 \pm 1.60$ \\
F & & 2.542 & 1.343 & 0.224 & 1.121 & 0.401 \\
P & & 0.081 & 0.274 & 0.802 & 0.334 & 0.672 \\
\hline
\end{tabular}

Notes: According to the kidney pathology results, there were 64 cases in the PMN group, 40 patients in the SMN group, and 60 patients in the non-MN group. There were no significant differences in clinical biochemical indexes among the three groups $(P>0.05)$.

Abbreviations: MN, membranous nephropathy; PMN, primary membranous nephropathy; SMN, secondary membranous nephropathy.

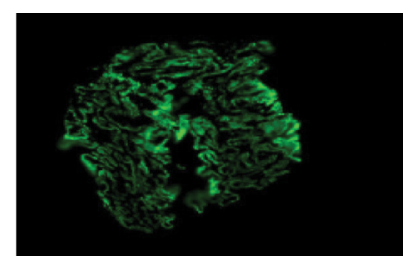

1A-PMN group

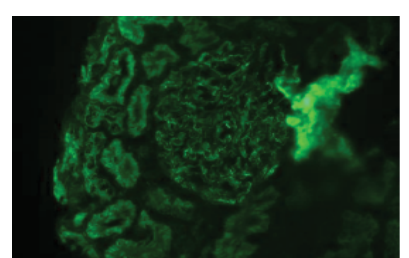

1B-SMN group

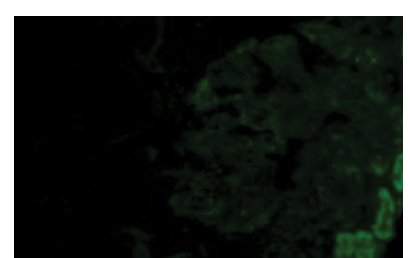

1C-non-MN group

Figure I Immunofluorescent staining of glomerular PLA2R $(\times 200)$.

Notes: The expression of PLA2R in glomeruli was significantly different among the three groups (AI-AC). Under immunofluorescence, deposition of a large number of immune complexes along the glomerular basement membrane in the PMN group, and deposition of a few or no immunolabeled complexes in the SMN group can be seen. No deposition of immune complexes was observed in the non-MN group.

Abbreviations: MN, membranous nephropathy; PMN, primary membranous nephropathy; SMN, secondary membranous nephropathy.

Table 2 Comparison of serum anti-PLA2R antibodies among the three groups

\begin{tabular}{|l|l|l|}
\hline Groups & $\mathbf{n}$ & Anti-PLA2R antibody $(\mathbf{R U} / \mathbf{m L})$ \\
\hline PMN & 64 & $226.62 \pm 366.49$ \\
SMN & 40 & $9.09 \pm 9.75^{\mathrm{a}}$ \\
non-MN & 60 & $5.11 \pm 3.80^{\mathrm{a}}$ \\
F & & 18.004 \\
P & & 0 \\
\hline
\end{tabular}

Notes: SMN and non-MN compared with $P M N$, ${ }^{a} P<0.05$. The serum anti-PLA2R antibody level in the PMN group was significantly higher than that in the SMN group and non-MN group.

Abbreviations: $\mathrm{MN}$, membranous nephropathy; $\mathrm{PMN}$, primary membranous nephropathy; SMN, secondary membranous nephropathy.

The serum anti-PLA2R antibody level in the PMN group was significantly higher than that in the SMN group and non-MN group. The serum anti-PLA2R antibody positivity rate in the PMN group was significantly higher than that in the SMN group and non-MN group $(P<0.01)$.

Serum anti-PLA2R antibody levels in the complete remission group, partial remission group, and non-remission group before and after treatment were measured.

The treatment programs of PMN is glucocorticoid combined with cyclophosphamide, or glucocorticoid combined with cyclosporine/FK506, and concurrently administered ACE inhibitor/angiotensin receptor blocker drugs. Before and
Table 3 Comparison of serum anti-PLA2R antibodies positive rate among the three groups

\begin{tabular}{|l|l|l|}
\hline Groups & $\mathbf{n}$ & $\begin{array}{l}\text { Serum anti-PLA2R antibody } \\
\text { positive rate example (\%) }\end{array}$ \\
\hline PMN & 64 & $87.5(56 / 64)$ \\
SMN & 40 & $20(8 / 40)^{\mathrm{a}}$ \\
non-MN & 60 & $0(0)^{\mathrm{a}}$ \\
$\chi^{2}$ & & 107.694 \\
$P$ & & 0 \\
\hline
\end{tabular}

Note: The serum anti-PLA2R antibody positivity rate in the PMN group was significantly higher than that in the $S M N$ group and non-MN group $\left({ }^{a} P<0.01\right)$.

Abbreviations: $\mathrm{MN}$, membranous nephropathy; PMN, primary membranous nephropathy; SMN, secondary membranous nephropathy.

after treatment, the serum levels of anti-PLA2R antibodies in the non-remission group were significantly higher than those in the complete remission group and partial remission group $(P<0.01)$. Before treatment, the serum anti-PLA2R antibody levels in the partial remission group and complete remission group were compared. The difference was not statistically significant $(P>0.05)$. After treatment, there was a statistically significant difference between the partial remission group and the complete remission group $(P<0.05)$. In the complete remission group and partial remission group, the antibody levels after treatment were lower than that before treatment $(P<0.01)$, while in the non-remission group, there 
was no significant difference in antibody levels after treatment compared with before treatment (Tables 4-6).

\section{Discussion}

PMN is a glomerular lesion caused by an antibody produced by the autoimmune system against normal glomerular epithelial cell antigens. Deposition of a large number of immune complexes activate the complement system to produce a membrane attack complex and promote changes to the extracellular matrix of the basement membrane by activating cytokines. ${ }^{13}$ PLA2 is a group of enzymes distributed in many

Table 4 Comparison of quantification levels of anti-PLA2R antibodies among three groups before treatment

\begin{tabular}{|l|l|l|}
\hline Groups & n & $\begin{array}{l}\text { Quantification levels of } \\
\text { anti-PLA2R antibodies } \\
\text { (RU/mL) }\end{array}$ \\
\hline Complete remission group & 16 & $122.31 \pm 71.98$ \\
Partial remission group & 24 & $198.11 \pm 219.00$ \\
Non-remission group & 16 & $483.42 \pm 630.48^{\mathrm{a}, \mathrm{b}}$ \\
F & & 6.404 \\
P & & 0.002 \\
\hline
\end{tabular}

Notes: Before treatment: non-remission group compared with complete remission group, ${ }^{a}<0.05$; non-remission group compared with the partial remission group, ${ }^{\mathrm{D} P}<0.01$. Before treatment, the serum levels of anti-PLA2R antibodies in the nonremission group were significantly higher than those in the complete remission group and partial remission group.

Table 5 Comparison of quantification levels of anti-PLA2R antibodies among three groups after treatment

\begin{tabular}{|l|l|l|}
\hline Groups & $\mathbf{n}$ & $\begin{array}{l}\text { Quantification levels of } \\
\text { anti-PLA2R antibodies } \\
\text { (RU/mL) }\end{array}$ \\
\hline Complete remission group & 16 & $6.89 \pm 5.76$ \\
Partial remission group & 24 & $12.74 \pm 9.18^{\mathrm{c}}$ \\
Non-remission group & 16 & $274.36 \pm 359.48^{\mathrm{a}, \mathrm{b}}$ \\
F & & 10.775 \\
P & & 0 \\
\hline
\end{tabular}

Notes: Compared with complete remission group, ${ }^{a} P<0.05, c P<0.05$; compared with the partial remission group, ${ }^{\mathrm{b} P}<0.01$. After treatment, the serum levels of anti-PLA2R antibodies in the non-remission group were significantly higher than those in the complete remission group and partial remission group; the serum levels of antiPLA2R antibodies in the partial remission group were higher than the complete remission group. organs of the human body. There are many subtypes of PLA2 that can bind to PLA2R. PLA2R is overexpressed in renal tissue epithelial cells of PMN patients. The expression of anti-PLA2R antibodies also follows, and a series of studies has confirmed that the levels of anti-PLA2R antibodies in PMN patients are significantly higher than those in normal and non-MN patients. ${ }^{14-17}$ The anti-PLA2R antibody has many biological characteristics and can cause complement system activation, podocyte injury, and basement membrane damage when combined with PLA2R on the glomerular podocyte membrane, ${ }^{18}$ leading to the emergence of large amounts of proteinuria.

This study showed that the quantitative serum antiPLA2R antibody level in the PMN group was $226.62 \pm 366.49$ $\mathrm{RU} / \mathrm{mL}$, and the antibody positive rate was $87.50 \%$. Whereas, the serum anti-PLA2R antibody quantification levels in the SMN group and non-MN group were 9.09 $\pm 9.75 \mathrm{RU} / \mathrm{mL}$ and $5.11 \pm 3.80 \mathrm{RU} / \mathrm{mL}$, respectively. The antibody positive rates for the SMN and non-MN groups were $25.00 \%$ and $0.00 \%$, respectively. The PMN group had a significantly higher proportion of patients who were serum anti-PLA2R antibody positive than did the SMN group and the non-MN group, these data show that serum anti-PLA2R antibody levels can accurately identify MN and non-MN, and the differential diagnosis of PMN and SMN is also very important. Clinically, for patients with contraindications to renal biopsy and patients who refuse to undergo renal biopsy, serum anti-PLA2R antibody measurement is feasible. Testing to improve the accuracy of PMN diagnostics makes noninvasive diagnosis of PMN possible. In addition, for patients with negative serum anti-PLA2R antibodies and pathological diagnosis of $\mathrm{MN}$, we must actively investigate and followup patients with or without secondary tumors, sarcoidosis, or SLE secondary factors to avoid misdiagnosis and missed diagnosis. Some PMN patients in the study had negative serum anti-PLA2R antibodies, the reason for this may be: 1) when serum samples were taken, $P M N$ was in remission or inactive. 2) In patients with PMN, there are other target antigens, such as THSD7A, and anti-THSD7A antibody is

Table 6 Comparison of quantification levels of anti-PLA2R antibodies before and after treatment

\begin{tabular}{|l|l|l|l|}
\hline Groups & $\begin{array}{l}\text { Complete remission } \\
\text { group }(\mathbf{n = 1 6 )}\end{array}$ & $\begin{array}{l}\text { Partial remission } \\
\text { group }(\mathbf{n = 2 4 )}\end{array}$ & $\begin{array}{l}\text { Non-remission } \\
\text { group }(\mathbf{n = 1 6})\end{array}$ \\
\hline Before treatment $(\mathrm{RU} / \mathrm{mL})$ & $122.31 \pm 71.98$ & $198.11 \pm 219.00$ & $483.42 \pm 630.48$ \\
After treatment $(\mathrm{RU} / \mathrm{mL})$ & $6.89 \pm 5.76^{\mathrm{a}}$ & $12.74 \pm 9.18^{\mathrm{a}}$ & $274.36 \pm 359.48$ \\
$t$ & 6.394 & 4.143 & 1.154 \\
$P$ & 0 & 0 & 0.26 \\
\hline
\end{tabular}

Notes: Compared with before treatment, ${ }^{a} P<0.05$. In the complete remission group and partial remission group, the antibody levels after treatment were lower than that before treatment, while in the non-remission group, there was no significant difference in antibody levels after treatment compared with before treatment. 
present in the patient's serum. ${ }^{19,20}$ This could act as another specific PMN antibody. 3) There may be secondary factors that have not yet been discovered. 4) Experimental error. The detection of serum anti-PLA2R antibody has the advantages of rapid, simple, sensitive, specific, and non-invasiveness for the auxiliary diagnosis of PMN. If combined with detection of serum anti-THSD7A antibodies, non-invasive diagnosis of PMN may be more sensitive and specific, this is also part of the content of our subsequent studies.

Patients with serum anti-PLA2R antibody-positive PMN were divided into complete remission, partial remission, and non-remission groups according to their condition after treatment. This study found that serum anti-PLA2R antibody quantification levels gradually decreased or even became negative with the remission of the disease. Therefore, the patient's serum anti-PLA2R antibody levels should be detected not only before treatment, but also during the entire course of PMN treatment. Dynamic monitoring of serum anti-PLA2R antibody quantitative level changes is a real-time reflection of the immune status in patients with PMN. Compared with the traditional approach of using proteinuria to determine disease severity and treatment efficacy, anti-PLA2R antibody is more effective and agile for monitoring the condition, predicting treatment efficacy, ${ }^{21}$ and determining the treatment time and duration. For example, monitoring of decreases in anti-PLA2R antibody quantitative levels can help to adjust the application of immunosuppressive agents to reduce and avoid immunosuppressive side effects, ${ }^{22}$ this is also one of the important values of this study. Domestic scholar Xh et al ${ }^{23}$ also considered that serum anti-PLA2R antibody levels are significantly related to the condition of $\mathrm{MN}$, and positive expression of anti-PLA2R antibodies is highly consistent with renal biopsy results. The dynamic monitoring of quantitative changes in serum anti-PLA2R antibodies can be used as an important reference indicator to reflect the immune status of PMN patients in real time. The level of anti-PLA2R antibody in serum may change with the development of the disease, but the expression of PLA2R in renal tissue is relatively stable, and the current study has not demonstrated any differences in the expression intensity of glomerular PLA2R and the prognosis of the patient's disease. In addition, there has been study showing that serum anti-PLA2R antibody test results before renal transplantation in patients with $\mathrm{MN}$ are helpful to predict the risk of recurrence after renal transplantation. ${ }^{24}$ Therefore, the detection of serum anti-PLA2R antibodies with regard to the timing of renal transplantation and renal evaluation has some significance, but such reports are few and further study is still needed.
The study of renal tissue PLA2R and serum anti-PLA2R antibodies has had an important impact on the diagnosis of PMN, making non-invasive diagnosis possible. At the same time, monitoring serum anti-PLA2R antibody quantitative levels can quantitatively reflect the immune status of PMN patients in real time. Monitoring the degree of disease activity of PMN patients, as well as drug efficacy, can help determine the timing of immunosuppressive therapy, which is conducive to improving clinical management. ${ }^{25}$ However, there are still many unsolved problems, such as the role of PLA2R and its antibodies in the pathogenesis of PMN; more large prospective and long-term follow-up studies are needed. The evaluation of serum anti-PLA2R antibody absolute levels and anti-PLA2R antibody quantification trends and determining which has more clinical significance related to the prognosis of PMN still needs further study. The combined detection of anti-PLA2R antibodies and antiTHSD7A antibodies will be more conducive to improving the diagnostic rate of PMN. Clinically, it has great application value and should be vigorously promoted. At the same time, future research should also focus on the pathogenesis of PMN that is negative for anti-PLA2R antibodies and anti-THSD7A antibodies. ${ }^{26}$

\section{Acknowledgment}

This work was supported by grants from the program of Natural Science Foundation of Higher Education of Anhui Province (KJ2015B021by and KJ2015B036by), the Key Program of Natural Science Foundation of Anhui Province (KJ2016A481), and the program of Natural Science Foundation of Bengbu Medical College (BYKY17101).

\section{Disclosure}

The authors report no conflicts of interest in this work.

\section{References}

1. Beck LH, Salant DJ. Membranous nephropathy: from models to man. J Clin Invest. 2014;124(6):2307-2314.

2. Couser WG. Primary Membranous Nephropathy. Clin JAm Soc Nephrol. 2017;12(6):983-997.

3. Yang Y, Zhang Z, Zhuo L, Chen DP, Li WG. The spectrum of biopsyproven glomerular disease in China: a systematic review. Chin Med J. 2018;131(6):731-735.

4. Debiec H, Ronco P. Immunopathogenesis of membranous nephropathy: an update. Semin Immunopathol. 2014;36(4):381-397.

5. Beck LH, Bonegio RG, Lambeau G, et al. M-type phospholipase A2 receptor as target antigen in idiopathic membranous nephropathy. N Engl J Med. 2009;361(1):11-21.

6. Radice A, Pieruzzi F, Trezzi B, et al. Diagnostic specificity of autoantibodies to M-type phospholipase A2 receptor (PLA2R) in differentiating idiopathic membranous nephropathy (IMN) from secondary forms and other glomerular diseases. J Nephrol. 2018;31(2):271-278. 
7. Zhang Q, Huang B, Liu X, et al. Ultrasensitive Quantitation of AntiPhospholipase A2 Receptor Antibody as A Diagnostic and Prognostic Indicator of Idiopathic Membranous Nephropathy. Sci Rep. 2017;7(1):12049.

8. Wu XX, Wen S, Zhu XJ. Diagnostic value of renal phospholipase A2 receptor and serum anti-phospholipase $\mathrm{A} 2$ receptor antibody in membranous nephropathy. J Central South Univ Med Sci. 2017;42(4):395-399.

9. Ramachandran R, Yadav AK, Kumar V, et al. Temporal Association Between PLA2R Antibodies and Clinical Outcomes in Primary Membranous Nephropathy. Kidney Int Rep. 2018;3(1):142-147.

10. Jiang W, Liu T, Dong H, et al. Relationship Between Serum DNA Replication, Clinicopathological Characteristics and Prognosis of Hepatitis B Virus-associated Glomerulonephritis with Severe Proteinuria by Lamivudine Plus Adefovir Dipivoxil Combination Therapy. Biomed Environ Sci. 2015;28(3):206-213.

11. Zambo I, Hermanova M, Adamkova Krakorova D, et al. Nestin expression in high-grade osteosarcomas and its clinical significance. Oncol Rep. 2012;27(5):1592-1598.

12. Kidney Disease: Improving Global Outcomes (KDIGO) CKD-MBD Update Work Group. KDIGO2017 Clinical Practice Guideline Update for the Diagnosis, Evaluation, Prevention, and Treatment of Chronic Kidney Disease -Mineral and Bone Disorder (CKD-MBD). Kidney Int Suppl. 2017;7:1-59.

13. Zhu H, Han Q, Wang Y, et al. The clinicopatholagical features of patients with membranous nephropathy. Int J Nephrol Renovasc Dis. 2018;11:33-40.

14. Guan Y, Li H, Duan L, Li Y, Wen YB, Li XW. Serum anti-phospolipase A2 receptor antibodies and glomerular IgG4 in the diagnosis of membranous nephropathy. Chin J Nephrol. 2015;31(3):198-202.

15. Zhu SS, Zhou SL, Zhou CM, Li YQ, Zou HQ. Detection of PLA2R1 in renal biopsy specimens of patients with idiopathic membranous nephropathy. J Southern Med Univ. 2015;35(4):526-529.

16. Cui J, Lou JY, Li WN, et al. The expression of PLA2R, anti-PLA2R and Nephrin in different types of membranous nephropathy. J Clin Pathol Res. 2015;35(4):628-634.
17. Ramachandran R, Kumar V, Singh N, et al. Utility of Determining Autoantibodies to M-type Phospholipase A2 Receptor in Diagnosing Primary Membranous Nephropathy: An Ideal Setting. Indian J Nephrol. 2017;27(5):413-415.

18. Mcquarrie EP. Anti-phospholipase A2 receptor antibodies in primary membranous nephropathy-10 key points. Nephrol Dial Transplant. 2018;33(2):212-213.

19. Han QX, Zhu HY. Clinical significance of serum PLA2R antibody and THSD7A antibody in idiopathic membranous nephropathy. Chin J Lab Med. 2017;40(8):564-568.

20. Seifert L, Hoxha E, Eichhoff AM, et al. The Most N-Terminal Region of THSD7A Is the Predominant Target for Autoimmunity in THSD7A-Associated Membranous Nephropathy. J Am Soc Nephrol. 2018;29(5):1536-1548.

21. Hoxha E, Thiele I, Zahner G, et al. Phospholipase A2 receptor autoantibodies and clinical outcome in patients with primary membranous nephropathy. J Am Soc Nephrol. 2014;25(6):1357-1366.

22. Wei SY, Wang YX, Li JS, et al. Serum Anti-PLA2R Antibody Predicts Treatment Outcome in Idiopathic Membranous Nephropathy. Am J Nephrol. 2016;43(2):129-140.

23. Xi XH, Li GF, Li X. [Serum PLA2R antibody in diagnosis and disease evaluation of patients with membranous nephropathy]. Chin J Modern Med. 2016;26(4):67-70. Chinese.

24. Gupta G, Fattah H, Ayalon R, et al. Pre-transplant phospholipase A2 receptor autoantibody concentration is associated with clinically significant recurrence of membranous nephropathy post-kidney transplantation. Clin Transplant. 2016;30(4):461-469.

25. Cattran DC, Brenchley PE. Membranous nephropathy: integrating basic science into improved clinical management. Kidney Int. 2017;91(3):566-574.

26. Floege J, Amann K. Primary glomerulonephritides. Lancet. 2016; 387(10032):2036-2048.

\section{Publish your work in this journal}

The International Journal of Nephrology and Renovascular Disease is an international, peer-reviewed open access journal focusing on the pathophysiology of the kidney and vascular supply. Epidemiology, screening, diagnosis, and treatment interventions are covered as well as basic science, biochemical and immunological studies. The manuscript management system is completely online and includes a very quick and fair peer-review system, which is all easy to use. Visit http://www. dovepress.com/testimonials.php to read real quotes from published authors. 